\title{
Clinical spectrum of females with HCCS mutation: from no clinical signs to a neonatal lethal form of the microphthalmia with linear skin defects (MLS) syndrome
}

Vanessa A van Rahden ${ }^{1}$, Isabella Rau', Sigrid Fuchs ${ }^{1}$, Friederike K Kosyna ${ }^{1,2}$, Hiram Larangeira de Almeida Jr ${ }^{3}$, Helen Fryssira ${ }^{4}$, Bertrand Isidor ${ }^{5,6}$, Anna Jauch $^{7}$, Madeleine Joubert ${ }^{8}$, Augusta M A Lachmeijer ${ }^{9}$, Christiane Zweier ${ }^{10}$, Ute Moog ${ }^{7}$ and Kerstin Kutsche ${ }^{1^{*}}$

\begin{abstract}
Background: Segmental Xp22.2 monosomy or a heterozygous HCCS mutation is associated with the microphthalmia with linear skin defects (MLS) or MIDAS (microphthalmia, dermal aplasia, and sclerocornea) syndrome, an X-linked disorder with male lethality. HCCS encodes the holocytochrome c-type synthase involved in mitochondrial oxidative phosphorylation (OXPHOS) and programmed cell death.

Methods: We characterized the X-chromosomal abnormality encompassing HCCS or an intragenic mutation in this gene in six new female patients with an MLS phenotype by cytogenetic analysis, fluorescence in situ hybridization, sequencing, and quantitative real-time PCR. The X chromosome inactivation (XCI) pattern was determined and clinical data of the patients were reviewed.

Results: Two terminal Xp deletions of $\geq 11.2 \mathrm{Mb}$, two submicroscopic copy number losses, one of $\sim 850 \mathrm{~kb}$ and one of $\geq 3 \mathrm{Mb}$, all covering HCCS, 1 nonsense, and one mosaic 2-bp deletion in HCCS are reported. All females had a completely (>98:2) or slightly skewed (82:18) XCl pattern. The most consistent clinical features were microphthalmia/ anophthalmia and sclerocornea/corneal opacity in all patients and congenital linear skin defects in 4/6. Additional manifestations included various ocular anomalies, cardiac defects, brain imaging abnormalities, microcephaly, postnatal growth retardation, and facial dysmorphism. However, no obvious clinical sign was observed in three female carriers who were relatives of one patient.
\end{abstract}

Conclusion: Our findings showed a wide phenotypic spectrum ranging from asymptomatic females with an HCCS mutation to patients with a neonatal lethal MLS form. Somatic mosaicism and the different ability of embryonic cells to cope with an OXPHOS defect and/or enhanced cell death upon HCCS deficiency likely underlie the great variability in phenotypes.

Keywords: HCCS, Microphthalmia, X-linked, Linear skin defects, X chromosome inactivation

\footnotetext{
* Correspondence: kkutsche@uke.de

'Institute of Human Genetics, University Medical Center Hamburg-Eppendorf, Martinistraße 52, 20246 Hamburg, Germany

Full list of author information is available at the end of the article
} 


\section{Background}

The microphthalmia with linear skin defects (MLS) syndrome (MIM 309801) is a rare X-linked neurodevelopmental disorder with male in utero lethality. The main clinical characteristics are uni- or bilateral microphthalmia and linear aplastic skin lesions which are usually limited to face and neck and develop into healed hyperpigmented areas with age. Additional features such as sclerocornea, corneal opacities, congenital heart defects, microcephaly, intellectual disability and agenesis of the corpus callosum have been observed less frequently [1].

The majority of MLS-affected patients carry a cytogenetically visible deletion or an unbalanced translocation leading to Xp22.2 monosomy [1]. After the minimal critical region of MLS syndrome had been defined to encompass the genes MID1, HCCS and ARHGAP6 in 1994 $[2,3]$, heterozygous intragenic mutations in $H C C S$ were identified as causative in 2006 [4]. Since then, only one additional HCCS missense mutation has been described in a sporadic female patient with microphthalmia and sclerocornea of both eyes [5]. HCCS encodes the holocytochrome $c$-type synthase that is involved in mitochondrial oxidative phosphorylation (OXPHOS) where it catalyzes the incorporation of heme moieties to cytochrome $c$ and cytochrome $c_{1}[6,7]$. Cytochrome $c_{1}$ is an integral component of complex III of the mitochondrial respiratory chain (MRC), while cytochrome $c$ functions as an electron shuttle between complexes III and IV [8]. Complementation studies in yeast revealed severely impaired OXPHOS upon HCCS deficiency [6]. Recently, mutations in another gene, $C O X 7 B$ in chromosome band $\mathrm{Xq} 21.1$, encoding a structural subunit of cytochrome $c$ oxidase (complex IV) involved in OXPHOS, have been identified in females with an MLS phenotype [9].

A high inter- and intrafamiliar phenotypic variability has been described in females with MLS syndrome [4,10-12]. Patients with an intragenic mutation or a (submicroscopic) deletion covering $H C C S$ can show the full-blown MLS phenotype associated with other anomalies, the classical combination of microphthalmia/anophthalmia and linear skin defects, isolated ocular manifestations, aplastic skin areas restricted to face and neck with no additional abnormalities or no symptoms at all $[4,5,10,11,13-15]$. As possible explanations for this great clinical variability somatic mosaicism for the mutation and the degree of skewed $\mathrm{X}$ chromosome inactivation (XCI) in different tissues have been discussed $[4,14,16,17]$.

Since 2007, we ascertained six novel female patients with a clinical diagnosis of MLS syndrome. Here we summarize the clinical and molecular data of these patients who were found to have different alterations involving the HCCS gene, ranging from classical chromosomal rearrangements of the $\mathrm{Xp} 22$ region to point mutations. We discuss different genetic mechanisms which protect females with an HCCS alteration from developing MLStypical clinical features.

\section{Methods \\ Patients}

The study was approved by the Ethics Committee of the Medical Chamber of Hamburg (No. PV3585). We obtained clinical data as well as blood, buccal swabs, lymphoblastoid cells and/or DNA samples from six patients with a clinical diagnosis of MLS syndrome, who were assessed by experienced clinical geneticists. The clinical data and samples were obtained with informed consent, including consent to use the photographs in this report.

\section{Cytogenetic and fluorescence in situ hybridization (FISH) analysis}

Conventional karyotyping was performed on metaphase spreads from peripheral blood lymphocytes by standard procedures. We used the Xp subtelomeric ToTelVysion probe (Abbott Molecular Inc, Des Plaines, IL, USA), the Vysis Steroid Sulfatase Deficiency probe (Vysis LSI STS; Abbott Molecular Inc) and the $\mathrm{X}$ centromere probe (Poseidon Satellite Enumeration Probe (SE) X (DXZ1), Kreatech Diagnostics, Amsterdam, NL) in FISH experiments. The bacterial artificial chromosome (BAC) clone RP11-163I1 (RPCI-11 human male BAC library) and fosmid clones (WIBR-2 human fosmid library [G248P8]) were received from the BACPAC Resource Center, Children's Hospital Oakland, CA, USA. BAC and fosmid DNA was prepared using the NucleoBond Xtra Midi kit (MachereyNagel, Düren, Germany). BAC and fosmid DNA was labeled by nick translation using the CGH Nick Translation Kit and Spectrum Green-dUTP and Spectrum Red-dUTP (Vysis, Downers Grove, IL, USA), respectively, according to the protocol provided. Chromosomes were counterstained using 4',6-diamidino-2-phenylindole (DAPI) (Serva Feinbiochemica, Heidelberg, Germany) and mounted in antifading solution (Vector Labs, Burlingame, CA, USA). Slides were analysed with a Leica Axioscope fluorescence microscope. Images were merged using a cooled CCD camera (Pieper, Schwerte, Germany) and CytoVision software (Applied Imaging, San Jose, CA, USA).

\section{Quantitative real-time polymerase chain reaction (qPCR)}

qPCR of HCCS exons on genomic DNA was carried out as described previously [18]. Primer sequences are available on request.

\section{Sequencing of HCCS}

DNA from whole blood, buccal swabs or lymphoblastoid cells was isolated by standard procedures. The coding region of the HCCS gene (exons 2-7; GenBank accession no. NM_005333.4) including flanking intronic sequences was amplified from genomic DNA. Primer sequences and 
PCR conditions are available on request. Amplicons were directly sequenced using the ABI BigDye Terminator Sequencing Kit (Applied Biosystems, Darmstadt, Germany) and an automated capillary sequencer (ABI 3500; Applied Biosystems). Sequence electropherograms were analysed using Sequence Pilot software (JSI Medical Systems, Kippenheim, Germany).

\section{Microsatellite analysis}

Where mutations were shown to have arisen de novo, we verified declared relationships by genotyping both parents and the patient at fifteen short tandem repeat (STR) loci and Amelogenin using the The AmpFLSTR Identifiler PCR Amplification Kit (Applied Biosystems). DNA isolated from lymphocytes and the lymphoblastoid cell line of patient 3 was genotyped using the The AmpFLSTR Identifiler PCR Amplification Kit.

\section{Cloning of a mutation bearing PCR product to analyse for mosaicism}

We cloned the amplicon of HCCS exon 6 in the pCR2.1 TOPO TA Cloning ${ }^{\bullet}$ Vector (Invitrogen, Karlsruhe, Germany). We picked bacterial clones and amplified them using standard protocols to identify those that contained a copy of the amplicon. We sequenced 138 PCR products to permit identification of those clones to contain mutation or wild-type bearing amplicons using the ABI BigDye Terminator Sequencing Kit (Applied Biosystems) and the automated capillary sequencer ABI 3500 (Applied Biosystems). Sequences were assembled and compared using the software SeqMan (DNASTAR, Madison, WI, USA).

\section{$\mathrm{X}$ chromosome inactivation analysis}

The methylation status of the $A R, P G K 1$ or $M A O A$ locus was examined by already described assays [19-21]. We modified the protocols as follows. For each DNA sample, two reactions were prepared. In the first reaction, $400 \mathrm{ng}$ of DNA was digested with $8 \mathrm{U}$ HpaII in a total volume of $10 \mu \mathrm{l}$ for $40 \mathrm{~min}$ at $37^{\circ} \mathrm{C}$. In the second reaction, the same amount of DNA was incubated with the reaction buffer but without restriction enzyme. The digested and undigested fractions were submitted to PCR using fluorochrome-coupled primers (AR: forward primer: 5'-[6FAM]CTTTCCAGAATCTGTTCCAG-3' and reverse primer: 5'-AAGGTTGCTGTTCCTCATC-3'; PGK1: forward primer: $5^{\prime}$-[6FAM]TGTTCCGCATTCTGCAAGC C-3' and reverse primer: 5' ${ }^{\prime}$-TATCCTTTTGTGCAGGAA CC-3'; MAOA: forward primer: 5'-AGTAATCCTTTCCA GCTGCCGAC-3' and reverse primer: 5' -[6FAM]TGCTTC ATAAAGGGATTCTCTTTG-3'). PCR conditions are available on request. For PGK1: PCR products were digested with $B s t \mathrm{XI}$ at $55^{\circ} \mathrm{C}$ for $3 \mathrm{~h}$ and the enzyme was inactivated at $65^{\circ} \mathrm{C}$ for $20 \mathrm{~min}$. For $M A O A$ : PCR amplicons were precipitated with ethanol and $3 \mathrm{M}$ $\mathrm{NaAc}$ ( $\mathrm{pH}$ 5.8), and the precipitate was dissolved in $\mathrm{H}_{2} \mathrm{O}$. After submitting the fraction to digestion with $\mathrm{SacI}$ at $37^{\circ} \mathrm{C}$ for $1 \mathrm{~h}$, the enzyme was inactivated at $65^{\circ} \mathrm{C}$ for $20 \mathrm{~min}$. The resulting amplification products ( $A R$ locus) or digested fractions (PGK1 and $M A O A$ loci) were run on an ABI 3500 automated sequencer and the peak areas were calculated by GeneMapper Software v4.1 (Applied Biosystems). To account for preferential allele amplification, values for the digested DNA were normalized with those for the undigested DNA of each proband. The XCI pattern (expressed arbitrarily as a ratio of the smaller: larger allele) was calculated by applying the previously reported formula [22]: skewing $=\left(\mathrm{d}_{1} / \mathrm{u}_{1}\right) /\left(\mathrm{d}_{1} / \mathrm{u}_{1}\right)+\left(\mathrm{d}_{2} / \mathrm{u}_{2}\right)$, where $d_{1}$ and $d_{2}$ represent the two raw peak area values of the digested sample, and $\mathrm{u}_{1}$ and $\mathrm{u}_{2}$ represent the raw peak area values of the undigested sample. In addition, one control male sample and one sample from a female known to have a completely skewed pattern of XCI (>98:2) were included in every batch of samples, to control for complete digestion and amplification efficiency.

$\mathrm{X}$-inactivation testing in patient 3 was initiated at the respective centre within routine diagnostics.

\section{Results}

In the six female patients with typical clinical manifestations of MLS syndrome, we identified heterozygous alterations of the HCCS gene. These comprised sequence-level mutations as well as microdeletions and cytogenetically visible deletions including multiple other genes (Table 1).

\section{Chromosomal analysis and FISH}

Routine cytogenetic analysis was performed in patients 1 and 3-6 diagnosed with MLS syndrome and revealed an apparently normal female karyotype in patients 1, 3, and 6 . Patients 4 and 5 showed a structurally abnormal karyotype with 46,X,del $(\mathrm{X})(\mathrm{p} 22)$ (Table 1). We confirmed the terminal Xp deletion by FISH using BAC RP11-163I1 encompassing the HCCS gene in patients 4 and 5 and a subtelomeric $\mathrm{Xp}$ probe together with a probe covering the STS gene (Xp22.32) in patient 4 (data not shown). The estimated minimum size of the two deletions was $\geq 11.2 \mathrm{Mb}$. FISH with the STS and X centromere probes on metaphase spreads of the parents of patient 4 revealed the expected number of signals (data not shown).

High resolution molecular karyotyping using Agilent Human Genome CGH 44 K oligonucleotide arrays (Agilent, Santa Clara, CA, USA) with the ISCA design (www. iscaconsortium.org) was performed on a clinical basis in patient 2 and revealed no disease-associated copy number variant (data not shown). 
Table 1 Collection of molecular and clinical data of 6 patients with MLS syndrome

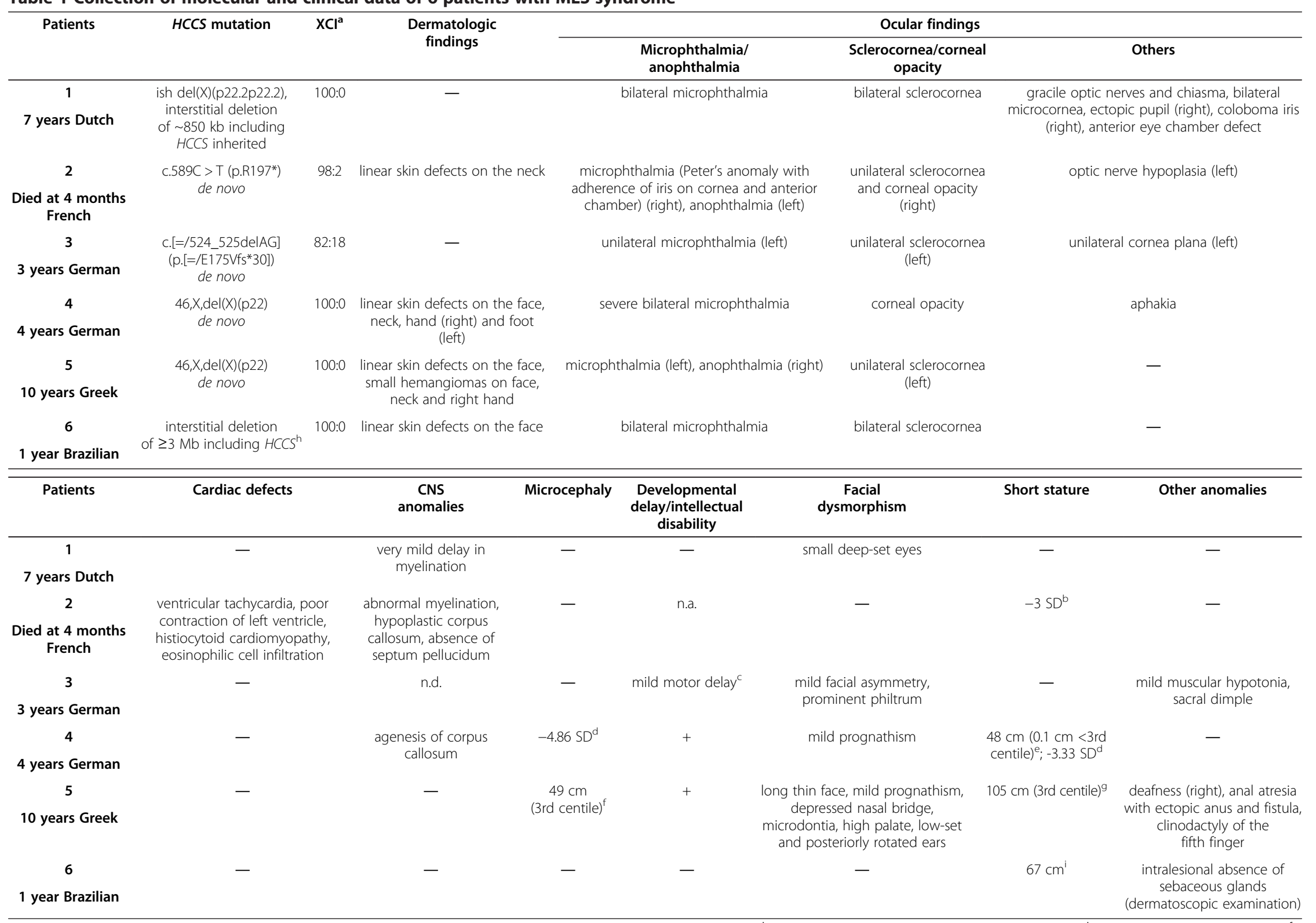

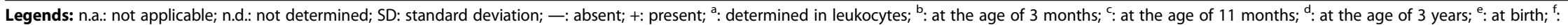

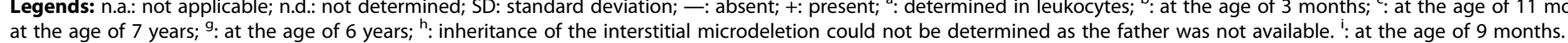




\section{Copy number analysis of HCCS by FISH and qPCR}

In patient 5 , the large Xp deletion was confirmed by qPCR of the selected HCCS exons 1, 3, 4, and 6 (Additional file 1: Figure S1). Expected values were observed for the relative copy number of the four HCCS exons in both parents of patient 5 (Additional file 1: Figure S1) indicating that her deletion occurred de novo.

To uncover a possible microdeletion covering HCCS, we performed FISH with RP11-163I1 and detected only one signal in patient 1 (III-1 in the pedigree of Figure 1A) suggesting an interstitial $\mathrm{Xp}$ deletion. We delineated the breakpoints by FISH with Xp22.2 fosmid clones (Figure 1B). For the distal deletion breakpoint, two signals were obtained for G248P86973A3, while only one signal was detected for G248P89648H11 (Additional file 1: Figure S2). To map the proximal deletion breakpoint, we used fosmid G248P82946A7 which gave only one signal, while G248P8046H9 yielded two signals (Additional file 1: Figure S2). Thus, patient 1 carried a $\sim 850 \mathrm{~kb}$ microdeletion covering the entire $H C C S$ gene and part of the neighboring genes MID1 and ARHGAP6 (ish $\operatorname{del}(\mathrm{X})$ (p22.2p22.2)) (Figure 1B and Table 1). qPCR confirmed that the relative copy number of $H C C S$ exons 2-7 in patient 1 was comparable to a haploid sample (Additional file 1: Figure S3). By FISH with BAC RP11-163I1 we detected the Xp22.2 microdeletion in the healthy mother (II-1 in Figure 1A) and the healthy maternal aunt of patient 1, too (II-2 in Figure 1A). In her healthy maternal grandmother, routine cytogenetic analysis revealed three $\mathrm{X}$ chromosomes in 25 analysed metaphases (data not shown). To confirm triple $\mathrm{X}$ syndrome, we performed FISH with RP11-163I1 in combination with the X centromere probe and detected two signals for the BAC and three for the centromere probe in 65 analysed metaphases (I-1 in Figure 1A and data not shown). However, in seven metaphases we found signals on two $\mathrm{X}$ chromosomes for the centromere probe and one signal for the BAC clone indicating mosaic trisomy $\mathrm{X}$ in patient's 1 maternal grandmother (data not shown). By analysing six $\mathrm{X}$-chromosomal microsatellite markers we confirmed the presence of three alleles in this female (data not shown). To summarize, patient's 1 healthy maternal grandmother carried the $\sim 850 \mathrm{~kb}$ microdeletion covering HCCS on one of her X chromosomes, and she had a mosaic form of triple X in her leukocytes (47,XXX.ish del (X)(p22.2p22.2)(HCCS-)[65]/46,XX.ish del(X)(p22.2p22.2) (HCCS-) [7]).

To analyse for the presence of a possible (submicroscopic) Xp22 deletion in patient 6 from Brazil, we had to perform qPCR experiments as only DNA and no lymphocyte suspension culture was available. The relative copy number of the selected HCCS exons 1, 3, 6 and 7 was found to be comparable with a haploid sample in patient 6 , while her mother yielded values comparable with a diploid sample (Figure 2A). A DNA sample of patient's 6 father was not available. To estimate the approximate size of the deletion, we selected genes located telomeric and centromeric to HCCS and performed additional qPCRs. We mapped the proximal breakpoint between the two genes TLR7 and RAB9A and the distal breakpoint between KAL1 and SHROOM2 (Figures 1B and 2B). The data demonstrates a submicroscopic interstitial $\mathrm{Xp}$ deletion of a minimum size of $3 \mathrm{Mb}$ in patient 6 encompassing the entire genes HCCS, MID1 and ARHGAP6 (Figure 1B).

\section{Sequence analysis of HCCS}

We Sanger-sequenced the coding exons 2-7 of HCCS in patients 2 and 3 . In patient 2 , we identified the heterozygous de novo nonsense mutation c.589C > T (p.R197*) in exon 6 (Figure 3A) (paternity confirmed; data not shown). In leukocyte-derived DNA of patient 3, we detected the 2bp deletion c.524_525delAG in exon 6 that results in a frameshift and introduction of a premature termination codon (p.E175Vfs*30) (Figure 3B). The sequence profile showed slightly lower signals for the mutant variant superimposed on the wild-type sequence suggesting that the mutation was present in the mosaic state (Figure 3B). To confirm somatic mosaicism of the 2-bp deletion in HCCS in patient 3, we amplified exon 6 from leukocyte-derived DNA, cloned the amplicon and subjected a total of 138 individual E.coli colonies to PCR followed by sequencing. We identified 102 wild-type alleles (73.9\%) and 36 alleles with the mutation c.524_525delAG (26.1\%) demonstrating somatic mosaicism in patient 3 (data not shown). Thus, patient 3 had a mixed population of in vivo lymphocytes: $52 \%$ of lymphocytes carry the heterozygous c.524_525delAG mutation and $\sim 48 \%$ carry two wild-type $H C C S$ alleles. We next investigated DNA from two other cell types of patient 3 and sequenced HCCS exon 6 in DNA isolated from buccal cells and a lymphoblastoid cell line (LCL). As shown in Figure 3B, the peak height of the two sequence profiles representing the two different $H C C S$ alleles in patient 3 was similar in buccal cell-derived DNA. In contrast, the sequencing pattern of the mutant HCCS allele is not any more visible in DNA isolated from LCLs (Figure 3B). Absence of the HCCS mutation in LCL-derived DNA suggested clonal evolution of the lymphoblastoid cell line of patient 3 [23,24]. By genotyping 16 genetic markers we confirmed that leukocyte- and LCL-derived DNA samples came from the same person (patient 3) (data not shown). Together, the data indicates that patient 3 carried a mosaic 2-bp deletion (c.[=/524_525delAG]/p.[=/E175Vfs*30]) in HCCS. Absence of the mutation in the parents is in line with somatic mosaicism in patient 3 (paternity confirmed; data not shown).

\section{$\mathrm{XCl}$ analysis}

The XCI pattern was determined in all patients. A totally (100:0) and extremely skewed (98:2) XCI was detected at 


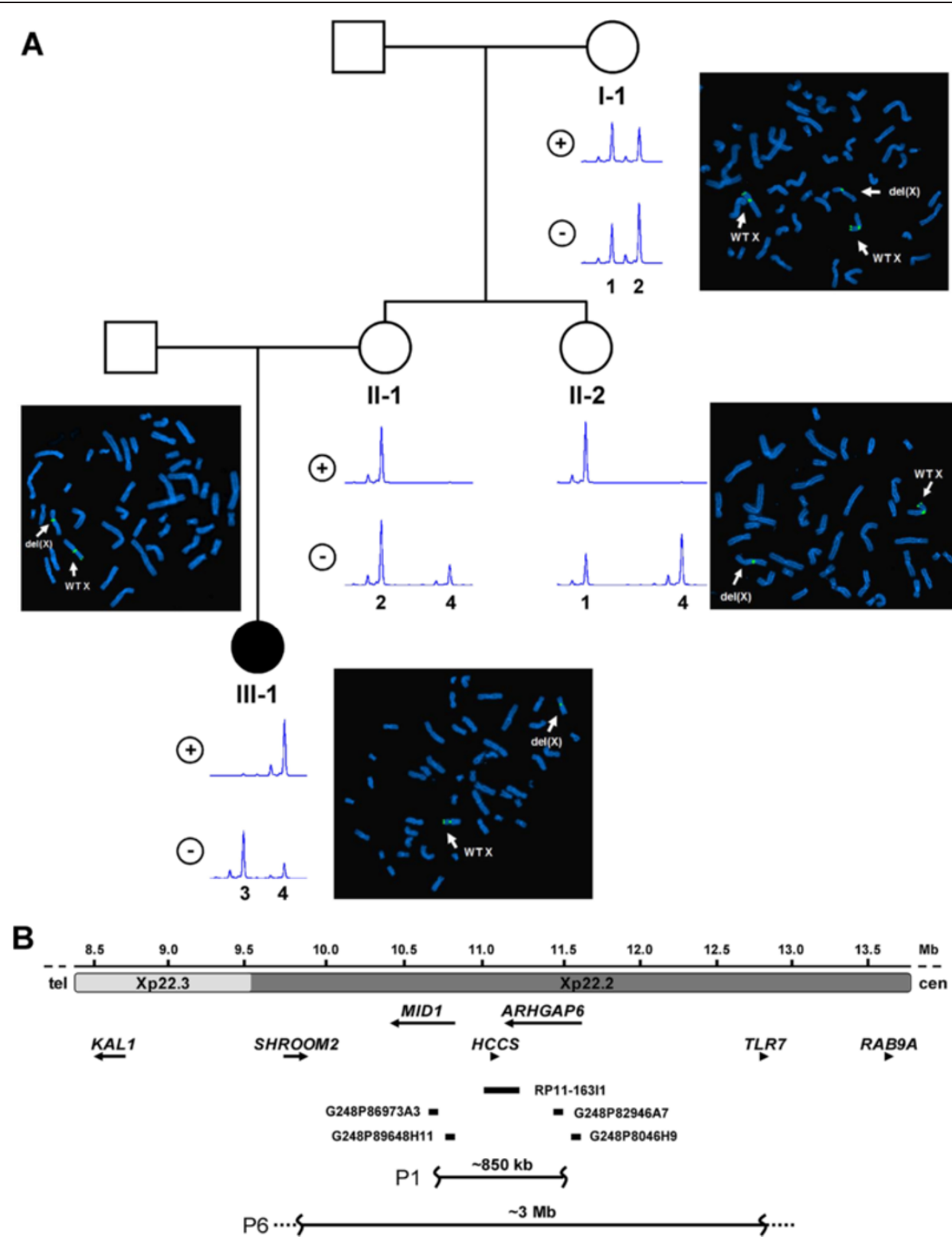

Figure 1 Pedigree, FISH and X chromosome inactivation of patient 1 and three healthy female relatives. A. Pedigree of patient's 1 family. Patient 1 (III-1) is affected by MLS syndrome, while her mother (II-1), her maternal aunt (II-2) and her maternal grandmother (I-1) are asymptomatic. FISH analysis with BAC RP11-163I1, spanning the HCCS gene on metaphase spreads of the four females revealed one signal in patient 1 (III-1), her mother (II-1) and her aunt (II-2). For the maternal grandmother (I-1), two signals for RP11-163l1 and three signals for the X centromere probe DXZ1 were obtained. BAC RP11-16311 and DXZ1 were labelled with Spectrum Green-dUTP. Arrows point to the wild-type X chromosome (WT X) and the $X$ chromosome with the microdeletion at Xp22.2 ( $\operatorname{del}(X))$. X chromosome inactivation was determined by analysing the methylation status of the androgen receptor gene at Xq12. Predigestion of genomic DNA isolated from lymphocytes with and without Hpall is indicated by (+) and $(-)$, respectively. Representative electropherograms show the different $A R$ alleles (designated as 1, 2, 3 and 4) in the four females. Females $\|-1\|-$,2 and III-1 have extremely skewed X inactivation (upper electropherograms indicated with +). B. Physical map of part of the Xp22.3 and Xp22.2 regions that are indicated by horizontal grey bars; $\mathrm{Mbs}$ and the telomere (tel) to centromere (cen) orientation are given. Arrows represent selected genes in Xp22 and gene symbols are given; arrowheads indicate the $5^{\prime} \rightarrow 3^{\prime}$ transcription direction of the genes. BAC RP11-16311 (RP11 Human BAC Library) and four Xp22.2 fosmid (WIBR-2 Human Fosmid Library) clones are indicated by black bars and names are given. Interstitial deletions found in patients 1 (P1) and 6 (P6) are depicted as horizontal black lines and the size of each deletion is given. Dotted and wavy lines indicate that the deletion breakpoints were not fine-mapped.

the $A R$ locus in leukocyte-derived DNA of patients 1 and 6 and patient 2, respectively (Table 1). As the interstitial Xp22.2 deletion in patient 1 was also found in three healthy family members, we also determined their XCI pattern and found complete skewing (100:0) in leukocytes of the mother and maternal aunt at the $A R$ locus 

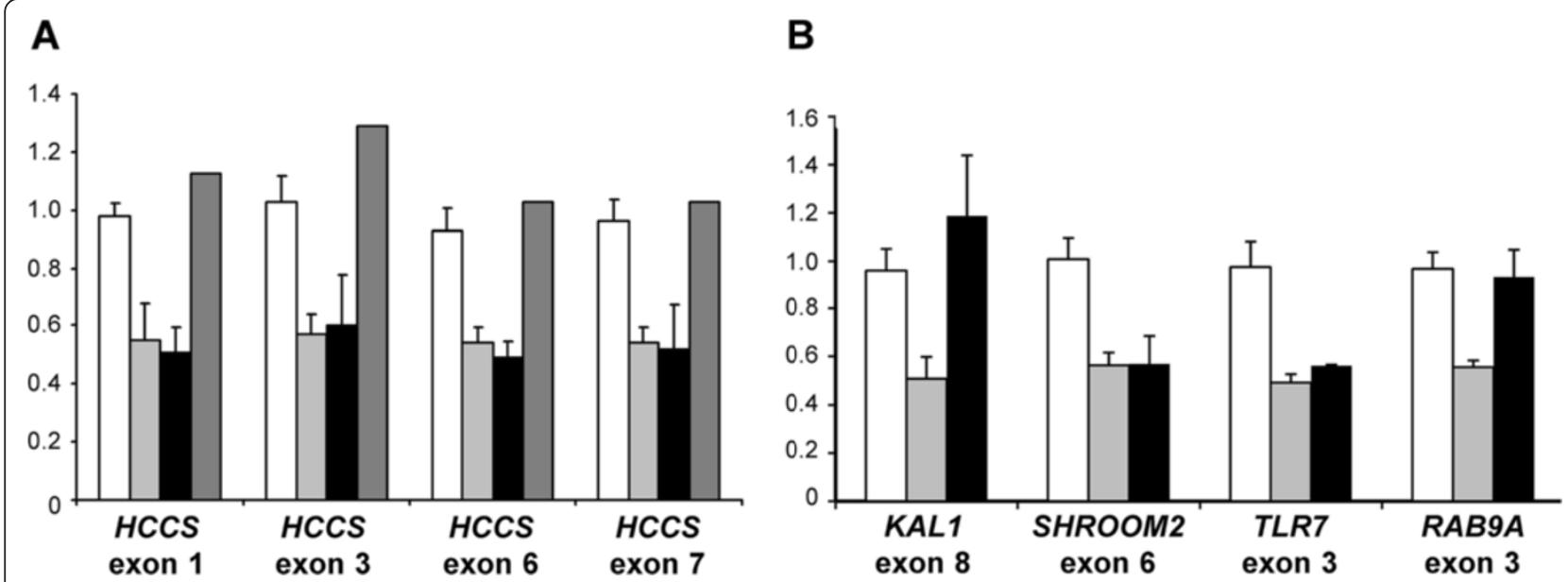

Figure 2 Copy number analysis of HCCS and neighboring genes by quantitative real-time PCR in patient 6 . A. Relative quantification of copy number of HCCS exons 1, 3, 6 and 7 by qPCR on genomic DNA of patient 6 (black bars) and her mother (darkgrey bars) revealed values that are comparable to a haploid sample (lightgrey bars) and a diploid sample (white bars), respectively. White, lightgrey and black bars represent the mean \pm SD of two independent experiments, each performed in duplicate. The darkgrey bars represent the mean of one experiment performed in duplicate for each exon. B. Relative quantification of copy number of HCCS surrounding genes in patient 6. qPCR for KAL1 exon 8 and RAB9A exon 3 on genomic DNA of patient 6 (black bars) revealed values that were comparable to a diploid sample (white bars), while those for SHROOM2 exon 6 and TLR7 exon 3 (black bars) were comparable with a haploid sample (lightgrey bars). Each bar represents the mean \pm SD of at least two experiments performed in duplicate.

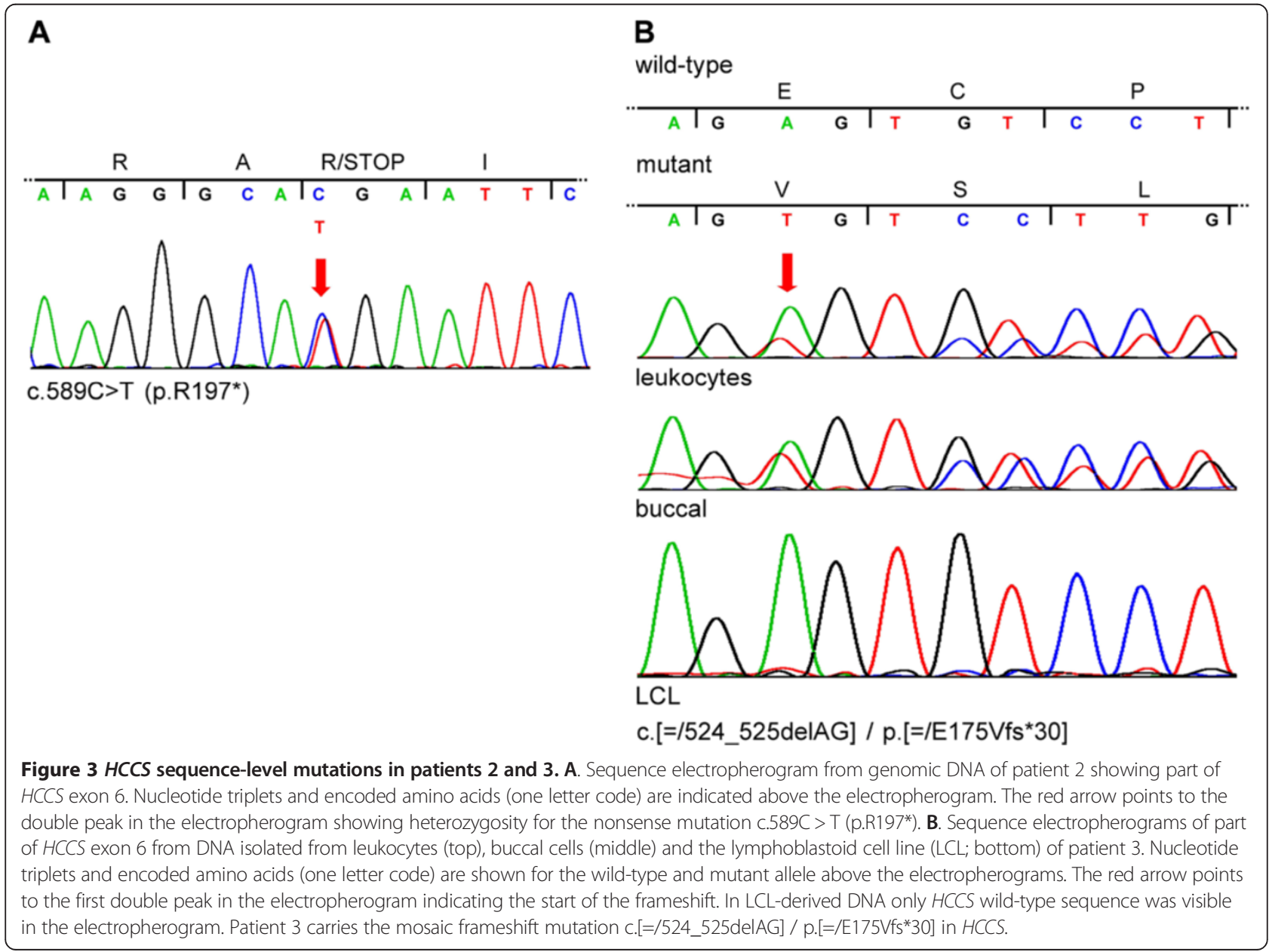


(Figure 1A). In the maternal grandmother, we detected two X-chromosomal alleles prior and after restriction of the DNA with the methylation sensitive enzyme HpaII (Figure 1A) suggesting that two of the three $\mathrm{X}$ chromosomes carry the same $A R$ allele and cannot be distinguished. Thus, a skewed XCI ratio possibly escaped detection in this female. Patients 4 and 5 were not informative at the $A R$ locus. In patient 4 , total XCI skewing (100:0) was found at the MAOA locus (Table 1). Similarly, patient 5 had an XCI ratio of 100:0 at the $P G K$ locus (Table 1). A slightly skewed XCI pattern (82:18) was identified in leukocyte-derived DNA of patient 3 at the FMR1 locus that is in line with a mosaic HCCS mutation (Table 1).

\section{Clinical data}

Clinical data of the six index patients were reviewed (Table 1). All were females aged between 4 months and 7 years at latest follow-up. Patient 2 died at the age of 4 months because of ventricular tachycardia and showed a histiocytoid cardiomyopathy on post-mortem pathological examination. The most consistent clinical features of all patients were unilateral/bilateral microphthalmia/anophthalmia and unilateral/bilateral sclerocornea/corneal opacity (Figure 4A, C-E, H and I). Additional ocular anomalies were frequently observed (Table 1). Four girls (patients 2 and 4-6) had linear skin defects at least on the face and neck (Figure 4A-C, F-I). Patient 4 presented with severe erythematous linear skin lesions on her face after birth (Figure 4F). They healed with age and developed to hyperpigmented areas (Figure 4G and H). Patients 1 and 3 did not show congenital skin lesions (Figure 4D and E). No cardiac defects except for those described in patient 2 were reported. Brain imaging studies revealed delayed/abnormal myelination in patients 1 and 2 and corpus callosum agenesis/hypoplasia in patients 2 and 4. Occipital frontal circumference in the low normal range or microcephaly was observed in patients 4 and 5. Individuals 2, 4, 5 and 6 had postnatal growth retardation of varying degree. Other anomalies (sacral dimple, deafness, anal atresia with ectopic anus and fistula, clinodactyly of fifth finger, and lack of sebaceous glands) were only seen in single patients. Minor craniofacial dysmorphism was observed in patients 1, 3, 4 and 5 (Table 1 and Figure 4C, D, E, and H).

\section{Discussion}

We present molecular and clinical data of six new female patients with typical features of MLS syndrome and a deletion or mutation involving HCCS. MLS syndrome was first described more than 20 years ago as a disorder characterized by congenital linear skin lesions and microphthalmia $[25,26]$. Since then a total of 62 cases with this clinical diagnosis have been reported [1,27-31]. The majority of patients had a chromosomal abnormality resulting in monosomy for the Xp22.2 region. MLS syndrome mainly affects females, however, ten males with an XX karyotype and Y-chromosomal material or 46, $\mathrm{XY}$ and a mosaic inversion involving the band Xp22.2 are known [11,15,29,32-36]. Finally, HCCS turned out to be the culprit gene in Xp22.2 [4] and is implicated in all chromosomal rearrangements reported in MLS syndrome-affected individuals to date. The known intragenic HCCS mutations comprise an $8.6-\mathrm{kb}$ copy number loss of part of the gene and the three point mutations c.589C > T (p.R197*), c.649C > T (p.R217C) and c.475G > A (p.E159K) [4,5]. By complementing a Saccharomyces cerevisiae strain deficient for the HCCS orthologue Cyc3p, the three sequence-level variants in HCCS were found to be loss-of-function mutations affecting MRC $[4,5]$. Additional evidence for implication of mitochondrial dysfunction in MLS syndrome came from the discovery of $C O X 7 B$ as the second gene for this neurocutaneous disorder; it encodes a structural subunit of MRC complex IV [9]. Deficiency of the COX7B or HCCS orthologue in medaka was found to recapitulate the MLS phenotype and demonstrated an essential function of the MRC complexes III and IV in human development in general and central nervous system (CNS) development in particular $[9,37]$.

The HCCS mutations identified in the six patients reported here represent the full spectrum of genetic alterations leading to null alleles: two patients had the common terminal Xp deletion with a size of $\geq 11.2 \mathrm{Mb}$ and two females carried a submicroscopic interstitial deletion, one of $\sim 850 \mathrm{~kb}$ and the other of $3 \mathrm{Mb}$ as minimum size. The four deletions contain multiple genes including HCCS. Only three cryptic interstitial deletions covering HCCS have been reported in MLS-affected females so far: One of $>3 \mathrm{Mb}$ [13], a second of $3.6 \mathrm{Mb}$ [38] and a third of $185-220 \mathrm{~kb}$ [30]. The two remaining MLS-affected females carried de novo intragenic sequence changes, the nonsense mutation c.589C > T (p.R197*) which seems to represent a recurrent mutation [4], and the novel mosaic 2-bp deletion c.[=/524_525delAG] (p.[=/E175Vfs*30])]. Similar to other reports, we could not establish a genotypephenotype correlation and observed a high intra- and interfamilial phenotypic variability in patients carrying an $H C C S$ alteration [1].

Unilateral/bilateral microphthalmia/anophthalmia and sclerocornea/corneal opacity of one or both eyes were found in all patients (Table 1). Other ocular findings include a wide variety of anomalies, such as microcornea, coloboma, anterior chamber defect, optic nerve hypoplasia (this report) as well as retinal abnormalities, congenital glaucoma with total/peripheral anterior synechia, and cataract $[4,30]$. Neonatal linear skin defects were seen in four out of the six patients and varied in severity; patients 1 and 3 did not show any linear skin lesions or 


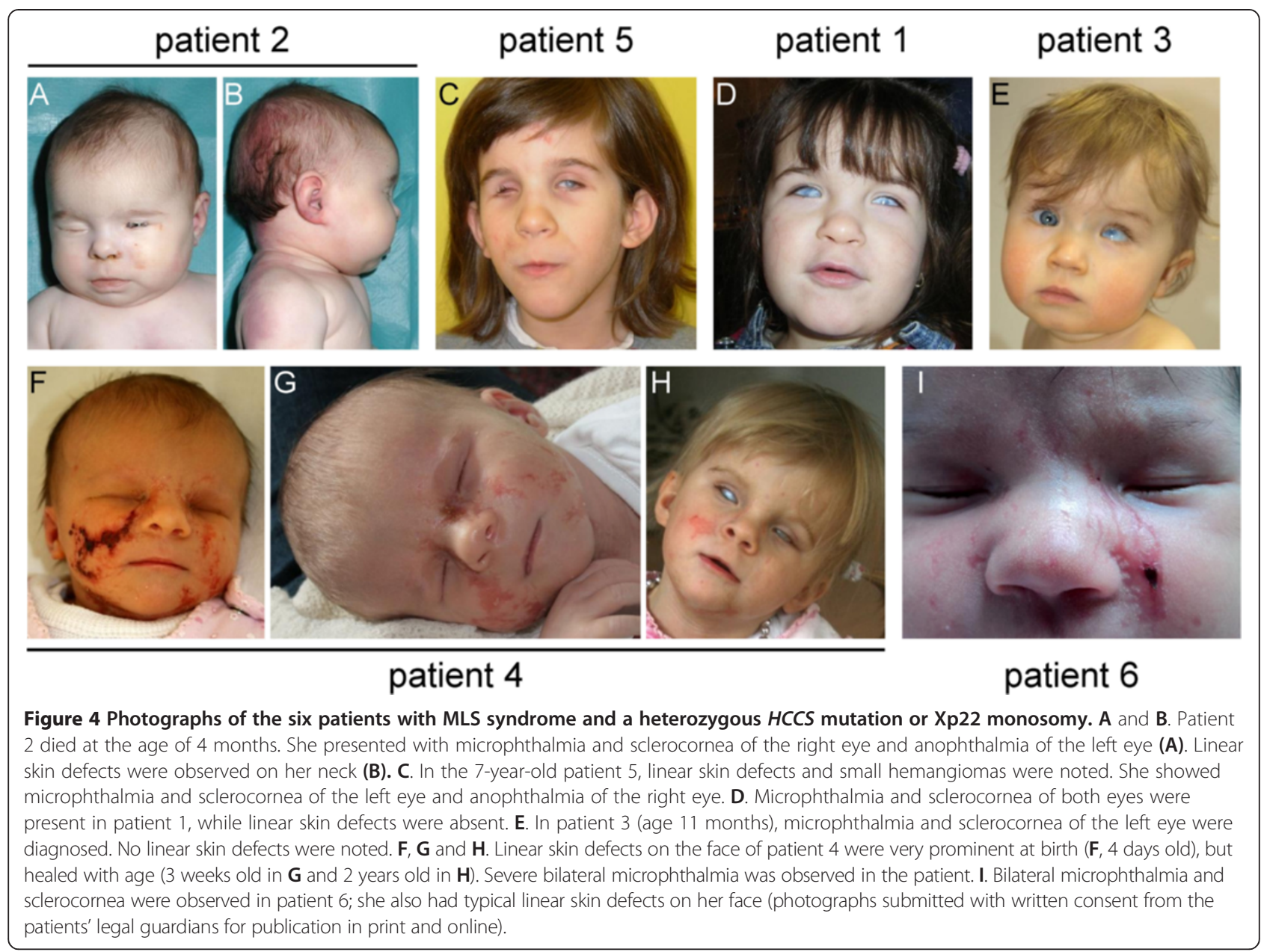

scars on their face and neck (Figure 4D and E). This observation is not unusual as a few reported individuals with Xp22 monosomy displayed eye abnormalities with absence of skin defects [33,34,39]. Similarly, in patients with a point mutation and small deletion in HCCS a variety of ocular anomalies without skin defects have been described $[4,5]$. To determine the phenotypic spectrum in MLS syndrome, we nowadays have to distinguish between $H C C S$ - and $C O X 7 B$-mutation positive patients. Interestingly, the four females with a $C O X 7 B$ mutation did not display microphthalmia/anophthalmia, but variable linear skin defects on the neck and face at birth [9]. Together, these data indicate that HCCS alterations cause variable eye and skin abnormalities, while the limited data on patients with a $C O X 7 B$ mutation suggest linear skin defects as the predominant feature.

We observed a wide range of additional clinical manifestations, including CNS anomalies such as abnormal myelination and hypoplasia/agenesis of corpus callosum and microcephaly. Cardiac defects comprise ventricular tachycardia, poor contraction of left ventricle, and histiocytoid cardiomyopathy. A few individuals had developmental delay, short stature and facial dysmorphism (Table 1). Rarely, hearing loss and anal atresia with ectopic anus and fistula, as seen in patient 5 have also been observed in other cases $[13,35,40,41]$. Patient 2 and one reported girl [4] carried the HCCS nonsense mutation c.589C > T (p.R197*). They show a remarkably similar full-blown MLS phenotype with microphthalmia, sclerocornea, linear erythrodermia, hypoplastic corpus callosum, and absence of septum pellucidum. However, patient 2 was more severely affected with anophthalmia and hypoplastic optic nerve of the left eye. She suffered from severe cardiac problems and died at age 4 months. Histiocytoid cardiomyopathy had been diagnosed, similar to a female infant with MLS syndrome who also died suddenly at 4 months of age [42].

The combination of clinical features in patient 2 clearly is at the severe end of the phenotypic spectrum in MLS syndrome. At the other end, no obvious MLStypical sign has been observed in the three HCCS-mutation positive female relatives of patient 1 . Although we 
cannot exclude the presence of mild linear skin lesions at birth in the mother, maternal aunt and grandmother of patient 1, non-penetrance in females with $\mathrm{HCCS}$ mutation or Xp22.2 monosomy has already been described $[4,14]$. In an Ashkenazi family, the index case and her sister showed eye anomalies and/or aplastic skin defects. The two sisters and their mother, who did not show any apparent sign of MLS carried the same small deletion in HCCS [4]. The same large terminal Xp deletion encompassing HCCS has been detected in a healthy female and her MLS-affected daughter, however, the mother was found to be a mosaic with $45, \mathrm{X}[11] / 46, \mathrm{X}, \mathrm{del}(\mathrm{X})(\mathrm{p} 22.2)$ [89] [14]. Thus, sex chromosome mosaicism may help to prevent the development of MLS-characteristic features in females and explain, at least in part, the high degree of inter- and intrafamiliar phenotypic variability $[11,14]$. In line with this, patient 3 who had unilateral ocular anomalies and no skin defects turned out to be a mosaic for a 2-bp HCCS deletion (c. [=/524_525delAG]). While this frameshift mutation was prominent in DNA from buccal cells, detailed analysis of leukocyte-derived DNA indicated a mixture of cells either carrying one wild-type and one mutant allele $(\sim 52 \%)$ or two wild-type alleles ( 48\%). Mosaicism was further confirmed by absence of the c.524_525delAG mutation in LCL-derived DNA of patient 3. For lymphoblastoid cell lines, rapid progression from polyclonality to pauciclonality or even monoclonality during cell culturing has been described [23,24]. Thus, clonal evolution likely accounts for disclosure of only HCCS wild-type sequence in DNA isolated from patient's 3 in vitro LCL culture (Figure 3B). Indeed, about $20 \%$ of established LCLs is affected by pauciclonality/monoclonality indicating that outgrowth of a single clone of fastgrowing B lymphocytes is a common phenomenon in cultured LCLs $[23,24]$. The apparently variable degree of mosaicism in different tissues of patient 3 might have contributed to her attenuated phenotype. Nevertheless, in a female infant with classical MLS and mosaic complex X-chromosomal rearrangements [43], mosaicism did not contribute to a mild phenotype indicating that other mechanisms account for the high clinical variability in females with HCCS null allele.

$\mathrm{XCI}$ has been discussed to contribute to phenotypic variability in MLS-affected females [17], including minor or no clinical signs. The $\mathrm{X}$ inactivation process starts about the time of late blastocyst or early gastrulation and inactivates one of the two $\mathrm{X}$ chromosomes, independent of the parental origin. The differential activity of the two $\mathrm{X}$ chromosomes is stably transmitted to all the descendants of a single cell and gives rise to cellular mosaicism in females [44]. Unequal inactivation of the parental alleles is known as skewing and can be the result of two different mechanisms. First, stochastic factors can cause non-random $\mathrm{XCI}$ in the early embryo, especially when the pool of precursor cells is limited. Secondary or acquired skewed XCI is the result of cell selection downstream of the $\mathrm{X}$ inactivation process $[44,45]$. In female carriers with Xp22 monosomy or an HCCS mutation, cells with an active aberrant $\mathrm{X}$ chromosome undergo severe respiratory problems due to disturbed mitochondrial OXPHOS as shown in yeast $[4,37]$. Impairment of the MRC is accompanied by a decrease in yeast chronological life span indicating that loss of HCCS negatively affects cell survival [37]. This finding suggests that cells with an active wild-type $\mathrm{X}$ chromosome reproduce faster and eventually outgrow cells expressing the HCCS mutation-bearing $\mathrm{X}$ chromosome leading to elimination of mutant cells and unbalanced/skewed XCI. Indeed, a remarkable regenerative capacity has been demonstrated in healthy cardiomyocytes of female mice with heart-specific inactivation of one Hccs gene copy. Hyperproliferation of healthy cardiac cells efficiently compensates for loss of $50 \%$ of $\mathrm{Hccs}$-deficient cells at a time after $\mathrm{XCI}$ has been completed to ensure the formation of a functional heart [46]. Thus, proliferative advantage of cells with the normal $\mathrm{X}$ chromosome active that leads to increased expansion of the normal cell population may ameliorate the effects of HCCS deficiency and decrease the likelihood of symptoms as also seen in female carriers of severe X-linked disorders and extremely skewed XCI, such as ATR-X and Wiscott-Aldrich syndrome [47,48]. Consequently, HCCS mutation-positive females with no apparent MLS sign, exemplified by the three healthy female relatives of patient 1 , are possibly the most favorable outcome of an effective overgrowth of cells expressing the normal X-linked allele. This demonstrates a great ability of the various tissues and organs to successfully eliminate HCCS-deficient dying cells during embryogenesis. In accordance with this, the cardiac phenotype in female mice with heart-specific Hccs deficiency primarily depends on the high proliferative capacity of the healthy cardiac cells (with the normal X chromosome active) [46].

Nevertheless, Xp22 monosomy or a heterozygous HCCS mutation bears the danger of developing MLS syndrometypical features raising the question "why is this so?". HCCS deficiency has been demonstrated to impair the MRC and induces overproduction of reactive oxygen species (ROS) [37]. The different ability of developing tissues and organs to cope with cells harbouring a defective OXPHOS system (as a result of an active mutant $\mathrm{X}$ chromosome) may account for the high clinical variability in MLS syndrome and could explain some specific clinical features, such as (histioctyoid) cardiomyopathy [49], agenesis of the corpus callosum [50], and deafness which are typically found in OXPHOS disorders $[8,51]$. In line with this, cardiomyopathy, ventricular dilation as well as various pathologies of the cardiac conduction system and sudden cardiac death 
detected in $\sim 40 \%$ of Hccs-deficient female mice have been explained by the amount and localization of residual diseased tissue in the heart at birth [46]. However, microphthalmia/anophthalmia, sclerocornea, microcephaly and linear skin defects are not usually found in mitochondrial diseases [8] and are unlikely to be caused by primary OXPHOS defects. Implication of mature cytochrome $c$, the final product of the holocytochrome $c$-type synthase (HCCS) reaction, in the mitochondria-dependent cell death pathway has led to the hypothesis that the inability of $\mathrm{HCCS}$-deficient cells to undergo cytochrome $c$ mediated apoptosis may direct cell death towards necrosis [4]. However, the recent discovery that MRC inhibition and enhanced ROS levels elicit a dramatic increase in caspase-dependent apoptosis in eyes and brain of hccsdeficient medaka fish may explain microphthalmia and CNS defects in MLS-affected patients [37]. The variable MLS phenotypes have been proposed to result from the different molecular responses of various tissues and organs on MRC impairment, enhanced ROS production and/ or increased cell death [37]. It has been hypothesized that the particular involvement of neural crest cells explains linear skin defects restricted to the face and neck $[11,25]$ and ocular anomalies. Indeed, extensive apoptosis leading to deficiency of neural crest cells has been shown to cause anophthalmia/microphthalmia upon influenza B virus infection during early chicken and mouse embryogenesis [52].

In summary, skewing of the XCI ratio in females with loss-of-function HCCS mutation represents the endpoint of a cell selection process during embryogenesis. These selection biases that occurred after primary XCI have been shown to similarly affect hematopoietic and epithelial cells suggesting that loss of some X-linked genes affects cellular growth in different cellular lineages in the same way [53]. The combination of (i) stochastic events during establishment of XCI, (ii) the genetic background, and (iii) secondary cell selection mechanisms comprising OXPHOS defects with or without enhanced cell death as the main effect has detrimental impact on specific developing organs/tissues and likely determines the phenotypic outcome in females with an HCCS null allele.

\section{Conclusions}

Mutation or copy number loss of HCCS is associated with a wide phenotypic spectrum ranging from no clinical signs to in utero lethality of MLS syndrome. Somatic mosaicism could be one factor contributing to a variable phenotype, however, cell selection mechanisms including OXPHOS defect and/or enhanced cell death upon HCCS deficiency likely underlie the great clinical variability in this rare neurocutaneous disorder.

\section{Additional file}

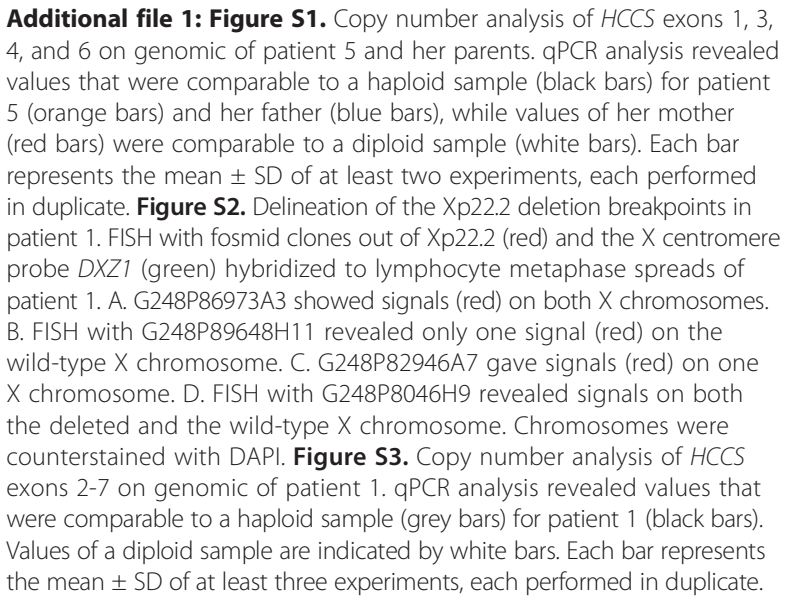

Competing interests

The authors declare that they have no competing interests.

\section{Authors' contributions}

HCCS molecular and FISH analysis: SF, AJ, KK, FKK, IR, VAvR; X chromosome inactivation analysis: IR; cytogenetic analysis: SF; patient ascertainment and clinical evaluation: HLA, HF, BI, MJ, AMAL, UM, CZ; manuscript writing: KK, UM, IR, VAvR; study design: KK. All authors contributed to and approved the final version of the paper.

\section{Acknowledgements}

We are grateful to the patients and their families who contributed to this study. We thank Inka Jantke and Dennis Zorndt for skilful technical assistance, Angela Fritsch, Birte Lübker and Fanny Kortüm for help with FISH experiments, and Cornelia Kraus for $\mathrm{XCl}$ analysis at the FMR1 locus. This work was supported by a grant from the Deutsche Forschungsgemeinschaft (KU 1240/6-1 to KK).

\section{Author details}

${ }^{1}$ Institute of Human Genetics, University Medical Center Hamburg-Eppendorf, Martinistraße 52, 20246 Hamburg, Germany. ${ }^{2}$ Current address: Department of Physiology, University of Lübeck, Lübeck, Germany. ${ }^{3}$ Federal and Catholic University of Pelotas, Pelotas, Brazil. ${ }^{4}$ Medical Genetics, School of Medicine, University of Athens, 'Aghia Sophia' Children's Hospital, Goudi, Athens, Greece. ${ }^{5}$ Unité de Génétique Clinique, CHU Nantes, Nantes, France. ${ }^{6}$ INSERM, UMR-S 957, Nantes, France. ${ }^{7}$ Institute of Human Genetics, Heidelberg University, Heidelberg, Germany. ${ }^{8}$ Department of Pathology, CHU Nantes, Nantes, France. ${ }^{9}$ Department of Clinical Genetics, VU University Medical Center, Amsterdam, The Netherlands. ${ }^{10}$ Institute of Human Genetics, Friedrich-Alexander-Universität Erlangen-Nürnberg, Erlangen, Germany.

Received: 30 January 2014 Accepted: 11 April 2014

Published: 15 April 2014

\section{References}

1. Morleo M, Franco B: Microphthalmia with Linear Skin Defects Syndrome In GeneReviews ${ }^{\mathrm{TM}}$. Edited by Pagon RA AM, Bird TD, Dolan CR, Fong CT, Stephens K. Seattle: University of Washington, Seattle; 2009.

2. Wapenaar MC, Bassi MT, Schaefer L, Grillo A, Ferrero GB, Chinault AC, Ballabio A, Zoghbi HY: The genes for X-linked ocular albinism (OA1) and microphthalmia with linear skin defects (MLS): cloning and characterization of the critical regions. Hum Mol Genet 1993, 2:947-952.

3. Wapenaar MC, Schiaffino MV, Bassi MT, Schaefer L, Chinault AC, Zoghbi HY Ballabio A: A YAC-based binning strategy facilitating the rapid assembly of cosmid contigs: $1.6 \mathrm{Mb}$ of overlapping cosmids in Xp22. Hum Mol Genet 1994, 3:1155-1161.

4. Wimplinger I, Morleo M, Rosenberger $G$, laconis D, Orth $U$, Meinecke $P$, Lerer I, Ballabio A, Gal A, Franco B, Kutsche K: Mutations of the 
mitochondrial holocytochrome c-type synthase in X-linked dominant microphthalmia with linear skin defects syndrome. Am J Hum Genet 2006, 79:878-889.

5. Wimplinger I, Shaw GM, Kutsche K: HCCS loss-of-function missense mutation in a female with bilateral microphthalmia and sclerocornea: a novel gene for severe ocular malformations? Mol Vis 2007, 13:1475-1482.

6. Schwarz QP, Cox TC: Complementation of a yeast CYC3 deficiency identifies an X-linked mammalian activator of apocytochrome c. Genomics 2002, 79:51-57.

7. Schaefer L, Ballabio A, Zoghbi HY: Cloning and characterization of a putative human holocytochrome c-type synthetase gene (HCCS) isolated from the critical region for microphthalmia with linear skin defects (MLS). Genomics 1996, 34:166-172.

8. Koopman WJ, Distelmaier F, Smeitink JA, Willems PH: OXPHOS mutations and neurodegeneration. EMBO J 2013, 32:9-29.

9. Indrieri A, van Rahden VA, Tiranti V, Morleo M, laconis D, Tammaro R, D'Amato I, Conte I, Maystadt I, Demuth S, Zvulunov A, Kutsche K, Zeviani M, Franco B: Mutations in COX7B cause microphthalmia with linear skin lesions, an unconventional mitochondrial disease. Am J Hum Genet 2012, 91:942-949.

10. Allanson J, Richter S: Linear skin defects and congenital microphthalmia: a new syndrome at Xp22.2. J Med Genet 1991, 28:143-144.

11. Lindsay EA, Grillo A, Ferrero GB, Roth EJ, Magenis E, Grompe M, Hulten M, Gould C, Baldini A, Zoghbi HY, Ballabio A: Microphthalmia with linear skin defects (MLS) syndrome: clinical, cytogenetic, and molecular characterization. Am J Med Genet 1994, 49:229-234.

12. Mücke J, Happle R, Theile H: MIDAS syndrome respectively MLS syndrome: a separate entity rather than a particular lyonization pattern of the gene causing Goltz syndrome. Am J Med Genet 1995, 57:117-118.

13. Morleo M, Pramparo T, Perone L, Gregato G, Le Caignec C, Mueller RF, Ogata T, Raas-Rothschild A, de Blois MC, Wilson LC, Zaidman G, Zuffardi O, Ballabio A, Franco B: Microphthalmia with linear skin defects (MLS) syndrome: clinical, cytogenetic, and molecular characterization of 11 cases. Am J Med Genet A 2005, 137:190-198.

14. Wimplinger I, Rauch A, Orth U, Schwarzer U, Trautmann U, Kutsche K: Mother and daughter with a terminal Xp deletion: implication of chromosomal mosaicism and X-inactivation in the high clinical variability of the microphthalmia with linear skin defects (MLS) syndrome. Eur J Med Genet 2007, 50:421-431

15. Kutsche K, Werner W, Bartsch O, von der Wense A, Meinecke P, Gal A: Microphthalmia with linear skin defects syndrome (MLS): a male with a mosaic paracentric inversion of Xp. Cytogenet Genome Res 2002, 99:297-302.

16. Van den Veyver IB: Skewed X inactivation in X-linked disorders. Semin Reprod Med 2001, 19:183-191.

17. Morleo M, Franco B: Dosage compensation of the mammalian X chromosome influences the phenotypic variability of $X$-linked dominant male-lethal disorders. J Med Genet 2008, 45:401-408.

18. Moog U, Kutsche K, Kortum F, Chilian B, Bierhals T, Apeshiotis N, Balg S, Chassaing N, Coubes C, Das S, Engels H, Van Esch H, Grasshoff U, Heise M, Isidor B, Jarvis J, Koehler U, Martin T, Oehl-Jaschkowitz B, Ortibus E, Pilz DT, Prabhakar P, Rappold G, Rau I, Rettenberger G, Schluter G, Scott RH, Shoukier M, Wohlleber E, Zirn B, et al: Phenotypic spectrum associated with CASK loss-of-function mutations. J Med Genet 2011, 48:741-751.

19. Allen RC, Zoghbi HY, Moseley AB, Rosenblatt HM, Belmont JW: Methylation of Hpall and Hhal sites near the polymorphic CAG repeat in the human androgen-receptor gene correlates with $\mathrm{X}$ chromosome inactivation. Am J Hum Genet 1992, 51:1229-1239.

20. van Kamp H, Jansen R, Willemze R, Fibbe WE, Landegent JE: Studies on clonality by PCR analysis of the PGK-1 gene. Nucleic Acids Res 1991, 19:2794

21. Hendriks RW, Chen ZY, Hinds H, Schuurman RK, Craig IW: An X chromosome inactivation assay based on differential methylation of a CpG island coupled to a VNTR polymorphism at the $5^{\prime}$ end of the monoamine oxidase A gene. Hum Mol Genet 1992, 1:662.

22. Hatakeyama C, Anderson $\mathrm{CL}$, Beever $\mathrm{CL}$, Penaherrera MS, Brown CJ, Robinson WP: The dynamics of X-inactivation skewing as women age. Clin Genet 2004, 66:327-332.

23. Plagnol V, Uz E, Wallace C, Stevens H, Clayton D, Ozcelik T, Todd JA: Extreme clonality in lymphoblastoid cell lines with implications for allele specific expression analyses. PLoS One 2008, 3:e2966.

24. Ryan JL, Kaufmann WK, Raab-Traub N, Oglesbee SE, Carey LA, Gulley ML: Clonal evolution of lymphoblastoid cell lines. Lab Invest 2006, 86:1193-1200.
25. Al-Gazali LI, Mueller RF, Caine A, Antoniou A, McCartney A, Fitchett M, Dennis NR: Two 46, $\mathrm{XX}, \mathrm{t}(\mathrm{X} ; \mathrm{Y})$ females with linear skin defects and congenital microphthalmia: a new syndrome at Xp22.3. J Med Genet 1990, 27:59-63.

26. Temple IK, Hurst JA, Hing S, Butler L, Baraitser M: De novo deletion of Xp22.2-pter in a female with linear skin lesions of the face and neck, microphthalmia, and anterior chamber eye anomalies. J Med Genet 1990, 27:56-58.

27. Carman KB, Yakut A, Sabuncu I, Yarar C: MIDAS (microphthalmia, dermal aplasia, sclerocornea) syndrome with central nervous system abnormalities. Clin Dysmorphol 2009, 18:234-235.

28. Garcia-Rabasco A, De-Unamuno B, Martinez F, Febrer-Bosch I, Alegre-de-Mique V: Microphthalmia with linear skin defects syndrome. Pediatr Dermatol 2013, 30:e230-e231.

29. Qidwai K, Pearson DM, Patel GS, Pober BR, Immken LL, Cheung SW, Scott DA: Deletions of Xp provide evidence for the role of holocytochrome C-type synthase (HCCS) in congenital diaphragmatic hernia. Am J Med Genet A 2010, 152A:1588-1590.

30. Vergult S, Leroy B, Claerhout I, Menten B: Familial cases of a submicroscopic Xp22.2 deletion: genotype-phenotype correlation in microphthalmia with linear skin defects syndrome. Mol Vis 2013, 19:311-318.

31. Zumwalt J, Moorhead C, Golkar L: Fourteen-month-old girl with facial skin thinning. Pediatr Dermatol 2012, 29:217-218

32. Anguiano A, Yang $X$, Felix JK, Hoo JJ: Twin brothers with MIDAS syndrome and XX karyotype. Am J Med Genet A 2003, 119A:47-49.

33. Kobayashi M, Kiyosawa M, Toyoura T, Tokoro T: An XX male with microphthalmos and sclerocornea. J Pediatr Ophthalmol Strabismus 1998, 35:122-124

34. Kono T, Migita T, Koyama S, Seki I: Another observation of microphthalmia in an XX male: microphthalmia with linear skin defects syndrome without linear skin lesions. J Hum Genet 1999, 44:63-68.

35. Paulger BR, Kraus EW, Pulitzer DR, Moore CM: Xp microdeletion syndrome characterized by pathognomonic linear skin defects on the head and neck. Pediatr Dermatol 1997, 14:26-30.

36. Stratton RF, Walter CA, Paulgar BR, Price ME, Moore CM: Second 46, XX male with MLS syndrome. Am J Med Genet A 1998, 76:37-41.

37. Indrieri A, Conte I, Chesi G, Romano A, Quartararo J, Tate R, Ghezzi D, Zeviani M, Goffrini P, Ferrero I, Bovolenta P, Franco B: The impairment of HCCS leads to MLS syndrome by activating a non-canonical cell death pathway in the brain and eyes. EMBO Mol Med 2013, 5:280-293.

38. Alberry MS, Juvanic G, Crolla J, Soothill P, Newbury-Ecob R: Pseudotail as a feature of microphthalmia with linear skin defects syndrome. Clin Dysmorphol 2011, 20:111-113.

39. Cape CJ, Zaidman GW, Beck AD, Kaufman AH: Phenotypic variation in ophthalmic manifestations of MIDAS syndrome (microphthalmia, dermal aplasia, and sclerocornea). Arch Ophthalmol 2004, 122:1070-1074.

40. Lindor NM, Michels W, Hoppe DA, Driscoll DJ, Leavitt JA, Dewald GW: Xp22.3 microdeletion syndrome with microphthalmia, sclerocornea, linear skin defects, and congenital heart defects. Am J Med Genet 1992, 44:61-65.

41. Steichen-Gersdorf E, Griesmaier E, Pientka FK, Kotzot D, Kutsche K: A severe form of the X-linked microphthalmia with linear skin defects syndrome in a female newborn. Clin Dysmorphol 2010, 19:82-84.

42. Bird LM, Krous HF, Eichenfield LF, Swalwell Cl, Jones MC: Female infant with oncocytic cardiomyopathy and microphthalmia with linear skin defects (MLS): a clue to the pathogenesis of oncocytic cardiomyopathy? Am J Med Genet 1994, 53:141-148.

43. Ogata T, Wakui K, Muroya K, Ohashi H, Matsuo N, Brown DM, Ishii T, Fukushima Y: Microphthalmia with linear skin defects syndrome in a mosaic female infant with monosomy for the Xp22 region: molecular analysis of the Xp22 breakpoint and the X-inactivation pattern. Hum Genet 1998, 103:51-56

44. Migeon BR: Why females are mosaics, X-chromosome inactivation, and sex differences in disease. Gend Med 2007, 4:97-105.

45. Minks J, Robinson WP, Brown CJ. A skewed view of X chromosome inactivation. J Clin Invest 2008, 118:20-23.

46. Drenckhahn JD, Schwarz QP, Gray S, Laskowski A, Kiriazis H, Ming Z, Harvey RP, Du XJ, Thorburn DR, Cox TC: Compensatory growth of healthy cardiac cells in the presence of diseased cells restores tissue homeostasis during heart development. Dev Cell 2008, 15:521-533. 
47. Migeon BR: The role of $X$ inactivation and cellular mosaicism in women's health and sex-specific diseases. JAMA 2006, 295:1428-1433.

48. Ørstavik $\mathrm{KH}: \mathrm{X}$ chromosome inactivation in clinical practice. Hum Genet 2009, 126:363-373.

49. Finsterer J: Histiocytoid cardiomyopathy: a mitochondrial disorder. Clin Cardiol 2008, 31:225-227.

50. Edvardson S, Porcelli V, Jalas C, Soiferman D, Kellner Y, Shaag A, Korman SH, Pierri CL, Scarcia P, Fraenkel ND, Segel R, Schechter A, Frumkin A, Pines O, Saada A, Palmieri L, Elpeleg O: Agenesis of corpus callosum and optic nerve hypoplasia due to mutations in SLC25A1 encoding the mitochondrial citrate transporter. J Med Genet 2013, 50:240-245.

51. Berardo A, Musumeci O, Toscano A: Cardiological manifestations of mitochondrial respiratory chain disorders. Acta Myol 2011, 30:9-15.

52. Chen BY, Chang HH, Chen ST, Tsao ZJ, Yeh SM, Wu CY, Lin DP: Congenital eye malformations associated with extensive periocular neural crest apoptosis after influenza $B$ virus infection during early embryogenesis. Mol Vis 2009, 15:2821-2828.

53. Bolduc V, Chagnon P, Provost S, Dube MP, Belisle C, Gingras M, Mollica L, Busque $L$ : No evidence that skewing of $X$ chromosome inactivation patterns is transmitted to offspring in humans. J Clin Invest 2008, 118:333-341.

doi:10.1186/1750-1172-9-53

Cite this article as: van Rahden et al:: Clinical spectrum of females with HCCS mutation: from no clinical signs to a neonatal lethal form of the microphthalmia with linear skin defects (MLS) syndrome. Orphanet Journal of Rare Diseases 2014 9:53.

\section{Submit your next manuscript to BioMed Central and take full advantage of:}

- Convenient online submission

- Thorough peer review

- No space constraints or color figure charges

- Immediate publication on acceptance

- Inclusion in PubMed, CAS, Scopus and Google Scholar

- Research which is freely available for redistribution 